\title{
Non-Neoplastic Anal Disorder
}

National Cancer Institute

\section{Source}

National Cancer Institute. Non-Neoplastic Anal Disorder. NCI Thesaurus. Code C53494.

A non-neoplastic disorder that affects the anus. Representative examples include hemorrhoids, ulcers, and infections. 